\title{
Stepwise Synthesis of Quercetin Bisglycosides Using Engineered Escherichia colis
}

\author{
Gyu Sik Choi, Hyeon Jeong Kim, Eun Ji Kim, Su Jin Lee, Youngshim Lee, and Joong-Hoon Ahn*
}

Department of Integrative Bioscience and Biotechnology, Bio/Molecular Informatics Center, Konkuk University, Seoul 05029, Republic of Korea

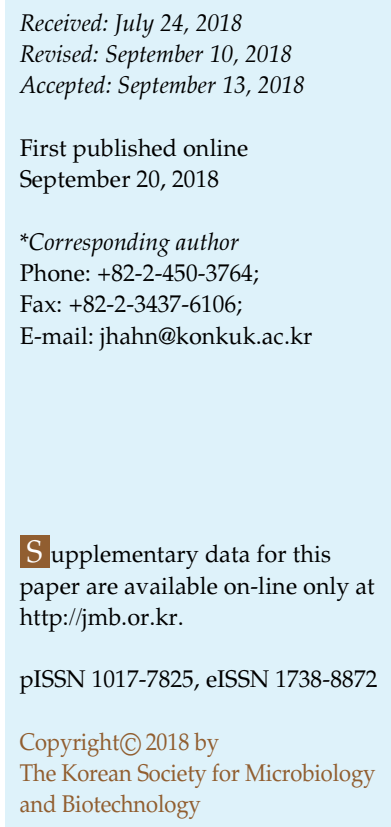

Synthesis of flavonoid glycoside is difficult due to diverse hydroxy groups in flavonoids and sugars. As such, enzymatic synthesis or biotransformation is an approach to solve this problem. In this report, we used stepwise biotransformation to synthesize two quercetin bisglycosides (quercetin 3-O-glucuronic acid 7-O-rhamnoside [Q-GR] and quercetin 3-Oarabinose 7-O-rhamnoside [Q-AR]) because quercetin $O$-rhamnosides contain antiviral activity. Two sequential enzymatic reactions were required to synthesize these flavonoid glycosides. We first synthesized quercetin 3-O-glucuronic acid [Q-G], and quercetin 3-Oarabinose [Q-A] from quercetin using E. coli harboring specific uridine diphopsphate glycosyltransferase (UGT) and genes for UDP-glucuronic acid and UDP-arabinose, respectively. With each quercetin 3-O-glycoside, rhamnosylation using E. coli harboring UGT and the gene for UDP-rhamnose was conducted. This approach resulted in the production of $44.8 \mathrm{mg} / 1 \mathrm{Q}-\mathrm{GR}$ and $45.1 \mathrm{mg} / 1 \mathrm{Q}-\mathrm{AR}$. This stepwise synthesis could be applicable to synthesize various natural product derivatives in case that the final yield of product was low due to the multistep reaction in one cell or when sequential synthesis is necessary in order to reduce the synthesis of byproducts.

Keywords: Bioconversion, flavonoid glycosides, glycosyltransferase

\section{Introduction}

Synthesis of glycones is a difficult task due to regioselectivity, and enzymatic synthesis could facilitate it. Glycosylation is carried out by uridine diphosphatedependent glycosyltransferases (UGTs) [1]. Most UGTs show substrate and regioselectivity, although some display promiscuity [2]. Flavonoids are one of the most abundant natural compounds [3], and flavonoid glycosides are common forms found in plants [4]. Thus, this is a good model compound to study regioselective synthesis using UGTs. In addition, bioconversion of flavonoids into flavonoid glycosides using Escherichia coli harboring UGT could be possible $[5,6]$, and the three-dimensional structure of flavonoid UGTs are available [2]. UGTs use nucleotide sugars (mostly UDP-sugar) as sugar donors and, therefore, have specificity for flavonoids and nucleotide sugar. Glucose, galactose, glucuronic acid, xylose, arabinose, and rhamnose are common sugars that are attached to flavonoids [7]. UGTs have been used as biocatalysts for the glycosylation of flavonoids, and various flavonoid glycosides, including flavonoid $O$-monoglycosides, flavonoid $O$-diglycosides, and flavonoid $O$-bisglycosides, have been synthesized in engineered E. coli $[5,8]$. For the synthesis of diverse flavonoid glycosides using E. coli, UGTs and nucleotide sugar biosynthetic genes were introduced. This approach achieved what could not be achieved through chemical synthesis.

E. coli synthesizes various nucleotide sugars, including UDP-glucoside, UDP-galactoside, UDP-glucuronic acid, UDP-N-acetylglucosamine, and TDP-glucoside [9]. These could serve as sugar donors for UGTs to synthesize flavonoid glycones. In addition, these nucleotide sugars can serve as substrates for the synthesis of other nucleotide sugars, such as UDP-arabinoside, UDP-xyloside, and UDPrhamnoside $[10,11]$. Therefore, E. coli is a good system to synthesize flavonoid glycosides. In addition, quercetin rhamnosides contain antiviral activity $[12,13]$. In this 
Table 1. Plasmids and E. coli strains used in this study.

\begin{tabular}{|c|c|c|}
\hline Plasmids or E. coli strain & Relevant properties or genetic marker & Source or reference \\
\hline \multicolumn{3}{|l|}{ Plasmids } \\
\hline pACYCDuet & P15A ori, $\mathrm{Cm}^{\mathrm{r}}$ & Novagen \\
\hline pCDFDuet & CDF ori, $\mathrm{Str}^{\mathrm{r}}$ & Novagen \\
\hline pETDuet & f1 ori, $A m p^{r}$ & Novagen \\
\hline pGEX 5X-2 & pBR322 ori, Amp ${ }^{r}$ & GE Healthcare \\
\hline pE-AmUGT-Ecugd & $\mathrm{pET}+$ UGT from A. majus + ugd from E. coli & [14] \\
\hline pG-D3 & pGEX 5X-2 + AUGT78D3 from A. thaliana & [10] \\
\hline pA-AtUXS-Ecugd & pACYC + UXS from A. thaliana $+u g d$ from E. coli & [10] \\
\hline pC-C1-RHM & pACYCD + AtUGT89C1 + RHM2 from A. thaliana & [15] \\
\hline pC-OsUXE & $\mathrm{pCDF}+$ UGE from Oryza sativa & [10] \\
\hline \multicolumn{3}{|l|}{ Strains } \\
\hline BL21 (DE3) & $\mathrm{F}^{-}$ompT hsdS $\mathrm{B}_{\mathrm{B}}\left(\mathrm{r}_{\mathrm{B}}^{-} \mathrm{m}_{\mathrm{B}}^{-}\right)$gal dcm lon (DE3) & Novagen \\
\hline B407 & BarnA harboring pG-D3, pA-AtUXS-Ecugd, and pC-OsUXE & [10] \\
\hline B509 & BarnA harboring pG-VvUGT and pA-Ecugd & [14] \\
\hline B-R & BL21 harboring pC-C1-RHM & [14] \\
\hline
\end{tabular}

report, we synthesized quercetin bisglycosides, Q-GR, and Q-AR. We used a stepwise synthesis method. First, quercetin 3-O-monoglycosides (quercetin 3-O-glucuronic acid [Q-G], and quercetin 3-O-arabinoside [Q-A]) were synthesized in E. coli. Subsequently, using this as a substrate, rhamnosylation was carried out in the second E. coli. Throughout this approach, $77.7 \mu \mathrm{M}$ Q-AR and $71.8 \mu \mathrm{M}$ Q-GR from $100 \mu \mathrm{M}$ quercetin were synthesized, respectively.

\section{Materials and Methods}

\section{Synthesis and Analysis of Metabolite}

Genes involved in Q-A synthesis (AtUGT78D3, OsUXE, AtUXS, and Ecugd) were cloned previously [10]. Genes for the synthesis of Q-G were cloned previously [14]. AtUGT89C1 and AtRHM2 were cloned and named pC-C1-Rham [15]. The E. coli strains used in this study are listed in Table 1.

The synthesis of Q-A and Q-G was carried out as described before [10, 14]. M9 medium containing antibiotics and $100 \mu \mathrm{M}$ quercetin was used for the synthesis of Q-A and Q-G. Proteins in the E. coli strain B-R grown in LB medium containing spectinomycin were induced by adding IPTG at the concentration of $1 \mathrm{mM}$ and incubating cells at $18^{\circ} \mathrm{C}$ for $30 \mathrm{~h}$. The cells were harvested and resuspended in the culture filtrate containing either $\mathrm{Q}-\mathrm{A}$ or $\mathrm{Q}-\mathrm{G}$, which was further incubated at $30^{\circ} \mathrm{C}$ with shaking. Optimal cell concentration of E. coli strain B-R was determined by adjusting the cell density to $\mathrm{OD}_{600}=1,2,3,4,5$, and 6 .

For the purification of the reaction product, the culture filtrate was passed through a column $(20 \times 3 \mathrm{~cm})$ packed with a non-polar copolymer styrene-divinylbenzene adsorbent resin (HP20, Samyang,
Korea). The binding reaction product was eluted with methanol and was evaporated to dryness. The sample was further purified using HPLC [16]. The structure of the product was determined using nuclear resonance spectroscopy (NMR) [15]. Complete assignments of the Q-GR and the Q-AR were in the supplementary data.

\section{Molecular Modeling of AtUGT89C1}

AtUGT89C1 was used to convert quercetin 3-O-glucoside and quercetin 3-O-rhamnoside into quercetin 3-O-glucoside 7-Orhamnoside and quercetin 3,7-O-dirhamnoside [15]. In order to test whether AtUGT89C1 could use Q-A and Q-R as a substrate, we carried out the molecular modeling. The model structure of AtUGT89C1 was obtained from the SWISS-MODEL server (https://swissmodel.expasy.org) by using the crystal structure of hydroquinone glucosyltransferase (PDB ID: 2vg8) as a template (sequence identity $=27.98 \%$ ). Uridine- $5^{\prime}$-diphosphate $($ UDP) in the template was merged into the model structure, and the UDP was modified into UDP-rhamnoside using the BUILD module of the MAESTRO program (Schrödinger Inc.). The resulting AtUGT89C1-UDP-rhamnoside complex was subjected to energy minimization to obtain a stable, low-energy conformation. Energy minimization was performed using the MacroModel module incorporated in the Maestro (Schrödinger Inc) (conjugate gradient minimization with 0.05 convergence criteria, the OPLS-AA force field, and GB/SA continuum water model). The ligand structures (Q-G and Q-A) were prepared using the BUILD module, which were energy-minimized through the MacroModel module incorporated in the Maestro (Schrödinger Inc). The energyminimized structure of AtUGT89C1-UDP-rhamnoside complex was used for ligand-docking in order to determine the binding 


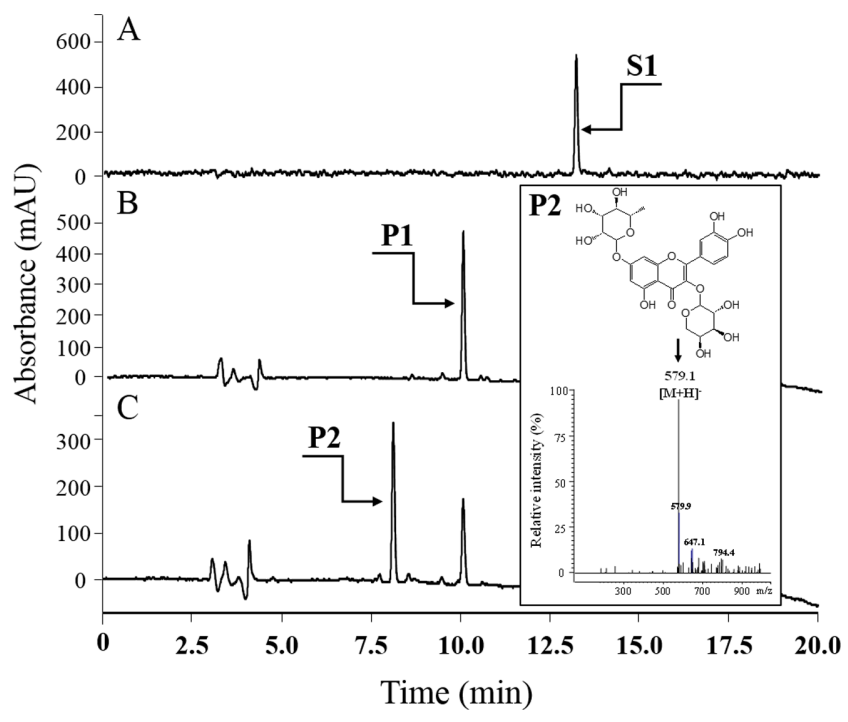

Fig. 1. Stepwise synthesis of quercetin 3-O-arabinoside-7-Orhamnoside.

A, standard quercetin; B, reaction product from strain B407 (P1); C, reaction product from strain $\mathrm{B}-\mathrm{R}$ fed the reaction product from strain B407. P1 is quercetin 3-O-arabinoside, and P2 is quercetin 3-Oarabinoside-7-O-rhamnoside. Inset is the mass spectra of the reaction product (P2).

mode of the flavonoid conjugates. We used the protein preparation utilities in Maestro to assign the charge state of ionizable residues, add hydrogens, and carry out energy minimization. The ligands were docked into the modeled AtUGT89C1-UDP-rhamnoside complex using GLIDE (http:/ / www.schrodinger.com). The default setting of the extreme precision mode of GLIDE was employed for the docking, and the top scored pose was chosen for the binding mode analysis.

\section{Results and Discussion}

\section{Synthesis of Quercetin O-Bisglycosides Using Engineered} E. coli

Quercetin is one of the abundant flavonoids in plants containing five hydroxy groups. This is a good substrate for the synthesis of quercetin O-bisglycosides. Quercetin $O$-bisglycosides were synthesized by two sequential glycosylation reactions. The first reaction was the attachment of sugar from UDP-glucuronic acid and UDParabinoside to the 3-OH group of quercetin. UGTs for each reaction were cloned from plants, and E. coli harboring each UGT and nucleotide sugar biosynthesis gene(s) were used to synthesize these quercetin 3-O-glycosides. For the synthesis of Q-A, the strain B407 [10] was used, and this strain harbored genes for the biosynthesis of UDParabinose from UDP-glucuronic acid and UGT by having a

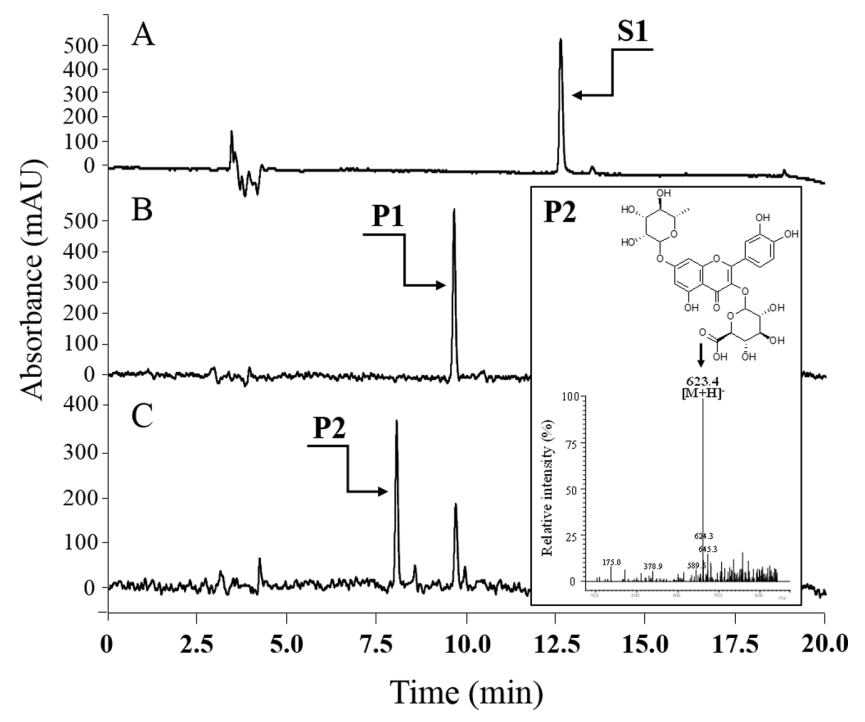

Fig. 2. Synthesis of quercetin 3-O-glucuronic acid 7-Orhamnoside.

A, standard quercetin (S1); B, reaction product from strain B509; C, reaction product from strain $B-R$ fed the reaction product from strain B509. P1 is quercetin 3-O-glucuronic acid, and P2 is quercetin 3-Oglucuronic acid 7-O-rhamnoside. Inset is the mass spectra of the reaction product.

high specificity for quercetin and UDP-arabinose. The B407 converted $100 \mu \mathrm{M}$ of quercetin into Q-A (Fig. 1B). The strain B509 [14], which harbored UGTs specific for quercetin and UDP-glucuronic acid and the ugd encoding UDP-glucose dehydrogenase that converts UDP-glucose into UDP-glucuronic acid, was used (Table 1). This strain also converted $100 \mu \mathrm{M}$ of quercetin into Q-G (Fig. 2B).

The second reaction used the Q-G or the Q-A as a sugar acceptor, and UDP-rhamnose as a sugar donor. AtUGT89C1 can use quercetin 3-O-glucoside and quercetin 3-Orhamnoside as a substrate and UDP-rhamnoside as a sugar donor [15]. However, it is unknown whether AtUGT89C1 could use Q-G or Q-A. In order to examine this, we conducted molecular docking using the modeled structure of AtUGT89C1. Each quercetin 3-O-glycoside along with UDP-rhamnose could fit into the active site of AtUGT89C1 (Fig. 3). The binding modes of Q-G or Q-A into AtUGT89C1 were very similar. Three amino acid residues (Ser152, Gln159, and Arg162) were key for binding to the sugar acceptor. Among them, Arg162 and Gln159 formed a hydrogen bond with hydroxy groups of sugar in Q-A or QG. Ser152 formed two hydrogen bonds with $3^{\prime}$ and $4^{\prime}$ hydroxy groups of quercetin. These results indicated that AtUGT89C1 can be used for the second glycosylation reaction. 
A

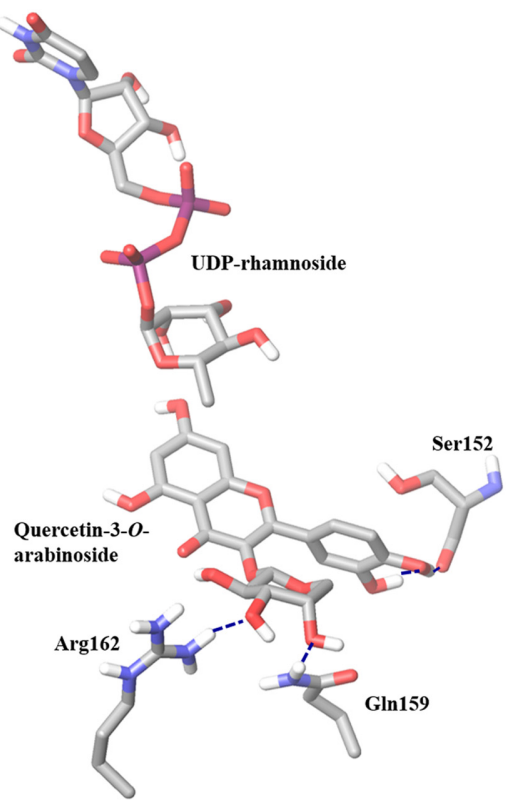

B

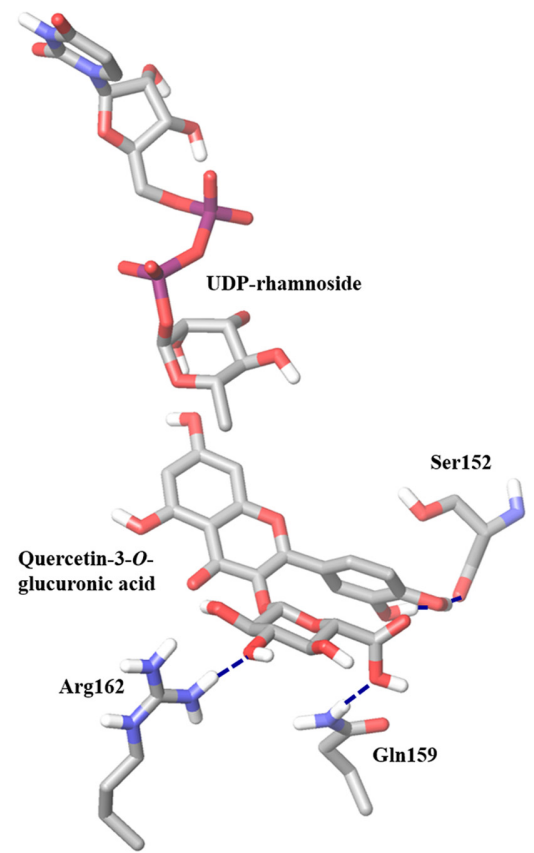

Fig. 3. Molecular docking of quercetin 3-O-arabinoside and UDP-rhamnoside (A) or quercetin 3-O-glucuronide and UDPrhamnoside (B) into AtUGT89C1.

Based on the molecular modeling result, we carried out the second reaction to synthesize quercetin $O$-bisglycosides. For the second glycosylation reaction, we used the strain BR, which contained AtUGT89C1 and RHM encoding rhamnose synthase, which converts UDP-glucose into UDP-rhamnose. In order to optimize the conversion of Q-A and Q-G into the corresponding 7-O-rhamnoside, the optimal initial cell concentration was examined. After protein induction in strain B-R by adding IPTG, the cells were harvested. The cells were resuspended with M9 medium at the concentration of 1, 2, 3, 4, 5, and 6 with $100 \mu \mathrm{M}$ of substrate. The conversion of each quercetin 3-Oglycoside into the corresponding quercetin $O$-bisglycoside increased until $\mathrm{OD}_{600}=5$. However, at $\mathrm{OD}_{600}=6$, the conversion decreased. Therefore, we used the cell concentration at $\mathrm{OD}_{600}=5$.

Culture filtrate of each quercetin 3-O-glycoside was mixed with the strain $B-R$ at $\mathrm{OD}_{600}=5$, and the production of quercetin $\mathrm{O}$-bisglycoside was monitored. Production of Q-AR was increased and reached to maximum at $36 \mathrm{~h}$, at which approximately $45.1 \mathrm{mg} / 1 \quad(77.7 \mu \mathrm{M})$ Q-AR was synthesized, and $6.7 \mathrm{mg} / 1(15.4 \mu \mathrm{M}) \mathrm{Q}-\mathrm{A}$ remained. The synthesis of Q-GR reached to the maximum at $24 \mathrm{~h}$, at which $44.8 \mathrm{mg} / 1(71.8 \mu \mathrm{M})$ was synthesized while $12.1 \mathrm{mg} / 1$ $(25.3 \mu \mathrm{M})$ of $\mathrm{Q}-\mathrm{G}$ remained. Starting from quercetin, $\mathrm{Q}-\mathrm{AR}$, and Q-GR showed $77.7 \%$ and $71.8 \%$ conversion rates, respectively.

\section{Structural Determination of Reaction Products}

The structures of quercetin O-bisglycosides were determined by NMR experiments. The purified Q-GR dissolved in DMSO- $d_{6}$, and ${ }^{1} \mathrm{H},{ }^{13} \mathrm{C},{ }^{1} \mathrm{H}-{ }^{1} \mathrm{H}$ COSY, ${ }^{1} \mathrm{H}-{ }^{1} \mathrm{H}$ NOESY, ${ }^{1} \mathrm{H}_{-}-{ }^{13} \mathrm{C} \mathrm{HMQC}$, and ${ }^{1} \mathrm{H}-{ }^{13} \mathrm{C}$ HMBC NMR experiments were performed. There were five aromatic signals in the ${ }^{1} \mathrm{H}$ NMR spectrum. They were easily assigned as protons of quercetin. Two ${ }^{1} \mathrm{H}$ signals at $5.55 \mathrm{ppm}$ and $5.47 \mathrm{ppm}$ were identified as H-R2 and H-G6, respectively. The chemical shifts of these two protons were distinguishable from other sugar protons because they were positioned between two oxygen atoms. Four NOE correlations, H-R2/H-6, H-R2/ H-8, H-G6/H-2', and H-G6/H-6' in the NOESY spectrum identified the sugar positions (Fig. 5A). The glucuronic acid was attached to $3-\mathrm{OH}$ while the rhamnose was attached to 7-OH via O-linked glycosylation. The ${ }^{1} \mathrm{H}$ of methyl from rhamnose showed at $1.12 \mathrm{ppm}$, and the other protons from glucuronic acid and rhamnose were dispersed from $3.28 \mathrm{ppm}$ to $3.85 \mathrm{ppm}$. From these, the structure of this compound was determined to be Q-GR.

${ }^{1} \mathrm{H},{ }^{13} \mathrm{C},{ }^{1} \mathrm{H}-{ }^{1} \mathrm{H}$ NOESY, ${ }^{1} \mathrm{H}-{ }^{13} \mathrm{C}$ HMQC, and ${ }^{1} \mathrm{H}-{ }^{13} \mathrm{C} \mathrm{HMBC}$ $\mathrm{NMR}$ experiments were also carried out with the purified 

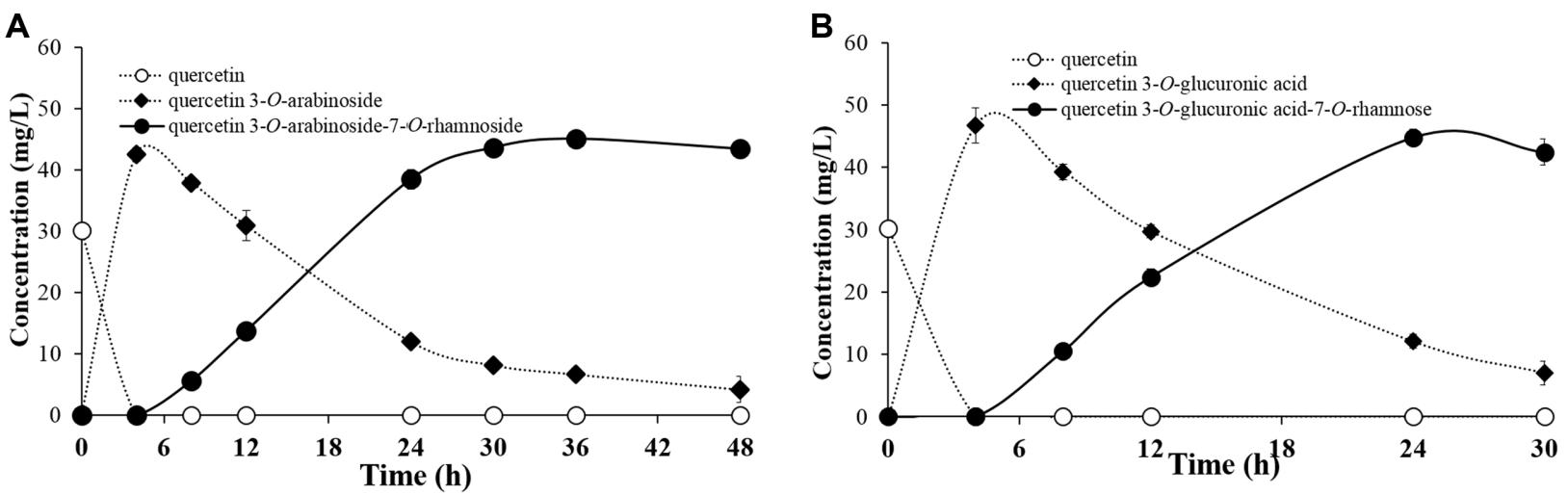

Fig. 4. Sequential synthesis of quercetin 3-O-arabinoside 7-O-rhamnoside (A) and quercetin 3-O-glucuronic acid 7-O-rhamnoside (B).

Quercetin 3-O-arabinoside and quercetin 3-O-glucuronic acid were synthesized using strain B407 and B509, respectively. Subsequently, the culture filtrate was mixed with strain B-R, and the resulting mixture was incubated for $48 \mathrm{~h}$.

product of putative Q-AR. All ${ }^{1} \mathrm{H}$ and partial ${ }^{13} \mathrm{C}$ peaks were assigned as mentioned above. Several important NOE and HMBC cross peaks were observed to confirm the structure (Fig. 5B). In the NOESY spectrum, H-2' and $\mathrm{H}-6^{\prime}$ correlated with $\mathrm{H}-\mathrm{A} 2$. H-A2 was also long-range coupled to the $\mathrm{C}-3$ of quercetin in the HMBC spectrum. The H-R2 showed NOEs with H-6 and H-8 and long-range coupling with $\mathrm{C}-7$ in the HMBC spectrum. These data supported that the synthesized compound was Q-AR.

We successfully synthesized Q-AR and Q-GR. Chemical synthesis of flavonoid $O$-bisglycosides has been carried out throughout multiple steps $[17,18]$. However, these two compounds have not been synthesized previously. Stepwise synthesis using two kinds of cells has an advantage over synthesis using one cell containing all genes (called a monoculture system). Synthesis using a monoculture system often produced byproduct(s). AtUGT89C1 showed less regioselectivity and substrate specificity. It could transfer a rhamnose group from UDP-rhamnose to $3-\mathrm{OH}$ of quercetin to form quercetin 3-O-rhamnoside. Subsequently, the quercetin 3-O-rhamnoside could be used for the second round rhamnosylation of AtUGT89C1 to form quercetin 3,7-O-bisrhamnoside [15]. However, the synthesis of quercetin 3-O-glycosides in one cell using regioselective UGT could prevent the formation of the byproducts as shown in this study. Recently, modulating cell ratios to consist of more than three different cells to synthesize anthocyanin has been attempted to maximize productivity [18]. This approach is called a polyculture system, which could reduce the byproduct formation and maximize the synthesis of the main product.

Due to the promiscuity of AtUGT89C1, it could be possible to synthesize other quercetin 3-O-glycoside 7-Orhamnoside. Previously, we showed the biosynthesis of
A

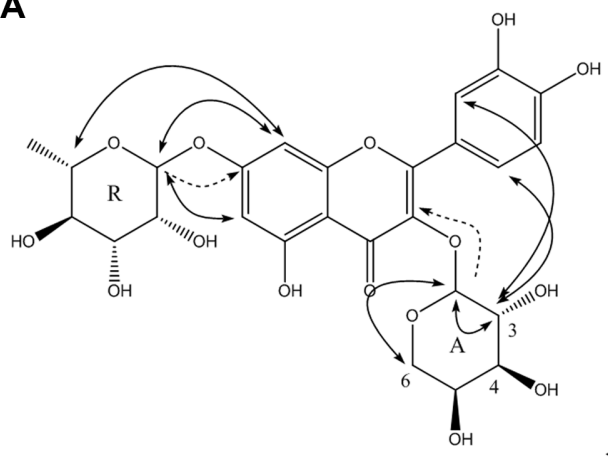

B

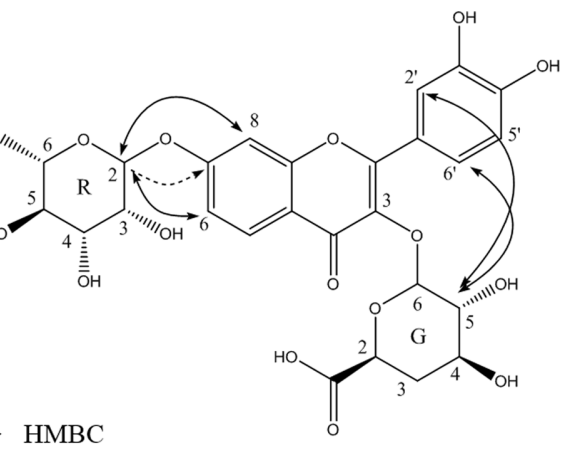

Fig. 5. Important long-range couplings and NOE correlations observed in the HMBC and NOESY spectra for the structure identifications of quercetin 3-O-arabinose 7-O-rahmnoside (A) and quercetin 3-O-glucuronic acid 7-O-rhamnoside (B). 
quercetin 3-O-glucoside 7-O-rhamnoside and quercetin $O$ 3,7-bisrhamnoside [15]. Quercetin 3-O-N-acetylglucosimine [20], quercetin 3-O-galactoside [14], and quercetin 3-Oxyloside [10] were synthesized previously. Molecular docking study of these three quercetin 3-O-glycosides showed a similar binding mode to quercetin 3-O-arabinose and quercetin 3-O-glucuronic acid (data not shown). Therefore, other flavonoid glycosides using AtUGT89C1 could be achievable.

\section{Acknowledgments}

This paper was supported by Konkuk University in 2016.

\section{Conflict of Interest}

The authors have no financial conflicts of interest to declare.

\section{References}

1. Jones P, Vogt T. 2001. Glycosyltransferases in secondary plant metabolism: tranquilizers and stimulant controllers. Planta 213: 164-174.

2. Osmani SA, Bak S, M?ller BL. 2009. Substrate specificity of plant UDP-dependent glycosyltransferase predicted from crystal structures and homology modeling. Phytochemitsry 70: 325-347.

3. Panche AN, Diwan AB, Chandra SR. 2016. Flavonoids: an overview. J. Nutr. Sci. 5: e47.

4. Veitch NC, Grayer RJ. 2011. Flavonoids and their glycosides, including anthocyanins. Nat. Prod. Rep. 28: 1626-1695.

5. Kim BG, Yang SM, Kim SY, Cha MN, Ahn J-H. 2015. Biosynthesis and production of glycosylated flavonoids in Escherichia coli: current state and perspectives. Appl. Microbiol. Biotechnol. 99: 2979-2988.

6. Han S-I, Lee J, Kim MS, Chung SJ, Kim J-H. 2017. Molecular cloning and characterization of a flavonoid glucosyltransferase from Byungkyool (Citrus platymamma hort. ex Tanaka). Appl. Biol. Chem. 60: 49-55.

7. Bowles D, Isayenkova J, Lim EK, Poppenberger B. 2005. Glycosyltransferases: managers of small molecules. Curr. Opin. Plant Biol. 8: 254-263.
8. Cho AR, An DG, Lee Y, Ahn J-H. 2016. Biotransformation of quercetin to quercetin 3-O-gentiobioside using engineered Escherichia coli. Appl. Biol. Chem. 59: 689-693.

9. Pabst M, Grass J, Fischl, R, Léonard R, Jin C, Hinterkörner G, et al. 2010. Nucleotide and nucleotide sugar analysis by liquid chromatography-electrospray ionization-mass spectrometry on surface-conditioned porous graphitic carbon. Anal. Chem. 82: $9782-9788$.

10. Han SH, Kim B-G, Yoon JA, Chong Y, Ahn J-H. 2014. Synthesis of flavonoid O-pentosides by Escherichia coli through engineering nucleotide sugar synthesis pathway and glycosyltransferase. Appl. Env. Microbiol. 80: 2754-2762.

11. Kim BG, Kim HJ, Ahn J-H. 2012. Production of bioactive flavonol rhamnosides by expression of plant genes in Escherichia coli. J. Agr. Food Chem. 60: 11143-11148.

12. Choi HJ, Kim JH, Lee CH, Ahn YJ, Song JH, Baek SH, et al. 2009. Antiviral activity of quercetin 7-rhamnoside against porcine epidemic diarrhea virus. Antiviral Res. 81: 77-81.

13. Song JH, Shim JK, Choi HJ. 2011. Quercetin 7-rhamnoside reduces porcine epidemic diarrhea virus replication via independent pathway of viral induced reactive oxygen species. Virol. J. 8: 460.

14. Kim SY, Lee HR, Park K-s, Kim BG, Ahn J-H. 2015. Metabolic engineering of Escherichia coli for the biosynthesis of flavonoid $\mathrm{O}$-glucuronides and flavonoid $\mathrm{O}$-galactoside. Appl. Microbiol. Biotechnol. 99: 2233-2242.

15. Kim HJ, Kim BG, Ahn J-H. 2013. Regioselective synthesis of flavonoid bisglycosides using Escherichia coli harboring two glycosyltransferases. Appl. Microbiol. Biotechnol. 97: 5275-5282.

16. Sim GY, Yang SM, Kim BG, Ahn J-H. 2015. Bacterial synthesis of $N$-hydroxycinnamoyl phenethylamines and tyramines. Microb. Cell Fact. 14: 162.

17. Urgaonkar S, Shaw JT. 2007. Synthesis of kaempferitrin. J. Org. Chem. 72: 4582-4585

18. Yang Y, Sun J, Yang Z, Han W, Zhang W-D, Yu B. 2012. Efficient synthesis of kaempferol 3,7-O-bisglycosides via successive glycosylation with glycosyl ortho-alkynylbenzoates and trifluoroacetimidates. Tetrahedron Lett. 53: 2773-2776.

19. Jones JA, Vernacchio VR, Collins SM, Shirke AN, Xiu Y, Englaender JA, et al. 2017. Complete biosynthesis of anthocyanins using E. coli polycultures. MBio 8: e00621-17.

20. Kim B-G, Sung SH, Ahn J-H. 2012. Biological synthesis of quercetin 3-O-N-acetylglucosamine conjugate using engineered Escherichia coli expressing UGT78D2. Appl. Microbiol. Biotechnol. 93: 2447-2453. 